\title{
Experimental study of the formation of inverted-V structures and their stratification using AUREOL-3 observations
}

\author{
O. Luízar ${ }^{1,2}$, M. V. Stepanova ${ }^{2}$, J. M. Bosqued ${ }^{3}$, E. E. Antonova ${ }^{4}$, R. A. Kovrazhkin ${ }^{5}$ \\ ${ }^{1}$ Departamento de Física, Universidad Nacional San Antonio Abad del Cusco, Peru \\ 2 Departamento de Física, Universidad de Santiago de Chile, Casilla 307, Santiago, Chile \\ ${ }^{3}$ Centre d'Etude Spatiale des Rayonnements, CNRS/UPS, BP 4346, 31028 Toulouse, France \\ ${ }^{4}$ Skobeltsyn Institute of Nuclear Physics, Moscow State University, Moscow 119899, Vorobievi Gori, Russia \\ ${ }^{5}$ Space Research Institute, Russian Academy of Sciences, Profsoyuznaya 84/32, Moscow 117810, Russia
}

Received: 17 November 1999 / Revised: 19 June 2000 / Accepted: 20 July 2000

\begin{abstract}
Multiple inverted-V structures are commonly observed on the same auroral zone crossing by a lowaltitude orbiting satellite. Such structures appear grouped and apparently result from an ionospheric and/or magnetospheric mechanism of stratification. More than two years of AUREOL-3 satellite observations were analyzed to study their properties and their formation in the framework of the ionosphere-magnetosphere coupling model proposed by Tverskoy. This model predicts some natural periodicity in the electrostatic potential profile (and subsequently in the field-aligned current profiles) that could account for oscillations experimentally observed in the auroral zone, such as successive inverted-Vs. Experimental results obtained during quiet or moderately active periods demonstrate that the number of structures observed within a given event is well described by a 'scaling' parameter provided by the hot plasma stratification theory and expressed in terms of the field-aligned current density, the total width of the current band, the plasma sheet ion temperature, and the heightintegrated Pedersen conductivity of the ionosphere. The latitudinal width, in the order of $100-200 \mathrm{~km}$ at ionospheric altitudes, is relatively independent of the current density, and is determined not only by the existence of a potential difference above the inverted-Vs, but also by basic oscillations of the ionosphere-magnetosphere coupling system predicted by Tverskoy. The large number of cases studied by the AUREOL-3 satellite provides reliable statistical trends which permits the validation of the model and the inference that the multiple structures currently observed can be related directly to oscillations of the magnetospheric potential (or the pressure gradients) on a scale of $\sim 1000-2000 \mathrm{~km}$ in the near-Earth plasma sheet. These oscillations arise in the Tverskoy model and may naturally result when the initial pressure gradients needed to generate a largescale field-aligned current have a sufficiently wide equatorial scale, of about $1 R_{E}$ or more.
\end{abstract}

Correspondence to: M. V. Stepanova

e-mail: mstepano@lauca.usach.cl
Key words: Magnetospheric physics (current systems; energetic particles, precipitating; magnetosphereionosphere interactions)

\section{Introduction}

Inverted-V (Frank and Ackerson, 1971) or Lambda (Heikkila, 1970) structures are the most prominent large-scale phenomena of auroral precipitation observed by low-altitude auroral satellites. During the last three decades considerable efforts have been concentrated on documenting their features and on understanding the mechanisms of their generation (see Lyons, 1981a, b; Lin and Hoffman, 1982; Bosqued et al., 1986). It is now well known that inverted-V structures correspond to the regions of intense upward field-aligned currents, carried by hot magnetospheric electrons which are accelerated at relatively low altitudes by field-aligned potential drops and which present shell-type distribution functions (Evans, 1974; Antonova and Tverskoy, 1975; Chiu and Schultz, 1978; Chiu et al., 1983; McFadden et al., 1999). According to Lyons (1981a, b), ionospheric electric fields under inverted-V structures must have a negative divergence. When the top of the acceleration region is located far from the Earth, the upward fieldaligned current (FAC) is linearly related to the fieldaligned potential drop (Knight, 1973; Antonova and Tverskoy, 1975; Fridman and Lemaire, 1980). However, in spite of these advances in understanding, the process of inverted-V structure formation remains unclear (see Borovsky, 1993 for a critical review), and we still need to know which plasma object in the magnetospheric equatorial plane maps into the ionosphere, forming an inverted-V structure (the problem of inverted-V roots).

The role of equatorial pressure gradients in the generation of the field-aligned currents and inverted-Vs has not yet clearly been evaluated. However, the contribution of pressure gradients to FAC generation during quiet time or at the onset of substorms has been 
quantitatively analyzed recently by a number of authors (e.g. Galperin et al., 1992; Galperin and Bosqued, 1999; Wing and Newell, 1998, 2000) by using the principal equation of perfect magnetosphere-ionosphere coupling for the most general case, corresponding to space scales comparable with the ion Larmor radius (Grad, 1964; Vasyliunas, 1970; Boström, 1975; Harel et al., 1981; Tverskoy, 1982a, b, 1983). In this approach the pressure gradient is balanced by Ampere's force, and at the same time inertial and viscous forces may be neglected when the large-scale bulk plasma velocity is lower than the Alfvén and sound velocities:

$$
\vec{\nabla} p=[\mathbf{j} \times \mathbf{B}]
$$

where $\mathbf{j}$ is the current density, and $\mathbf{B}$ is the magnetic field.

In the presence of the conducting ionosphere, in the stationary case the ionospheric part of the FAC (left side) is equal to the magnetospheric part (right side) and the fundamental equation of the magnetosphere-ionosphere interaction is (see, for a detailed derivation and analysis, Heinemann, 1990, Heinemann and Pontius, 1991):

$\operatorname{div}\left(\Sigma \vec{\nabla} U_{i}\right)=\mathbf{n}[\vec{\nabla} W \times \vec{\nabla} p]$

where $\Sigma$ is the height-integrated conductivity tensor, $U_{i}$ is the ionospheric potential, $W=\int \mathrm{d} l / B$ is the magnetic flux tube volume per unit flux, integrated from the equatorial plane to the ionosphere, $l$ is the length along the field line, $\mathbf{n}$ is the unit vector (along the field line) pointing towards the ionosphere, and $p$ is the scalar pressure. Upward FAC is assumed positive.

Antonova and Ganushkina (1997) have shown that the necessary condition for FAC generation is the noncoincidence of the current contours with the $W$ contours in the equatorial plane, according to the relation:

$\mathbf{j}_{\|}=-\frac{B_{i}}{B_{e}} \frac{\mathbf{B}_{e}}{B_{e}}[[\mathbf{j} \times \mathbf{B}] \times \vec{\nabla} W]$

where $B_{i}$ and $B_{e}$ are the magnetic fields, at the ionospheric altitudes and at the equatorial plane, respectively. In the inner region of the symmetric ring current, both contours are concentric circles and the FAC cannot be generated. In the geomagnetic tail the current flows across the tail, the contours of constant $W$ are dawn-to-dusk straight lines (around midnight) and the FAC cannot be generated. However, the generation of intense FACs is possible when and where a very small angle exists between $\mathbf{j}$ and $\vec{\nabla} W$ in the nightside transition region from a quasi-dipolar to a tail-like magnetic configuration (Galperin et al., 1992; Galperin and Bosqued, 1999). In this region, the plasma pressure reaches the maximum needed to generate the region 2 currents.

The role of the pressure gradients in the generation of the dawnside region 1 and region 2 currents has been analyzed by Iijima et al. (1997). This analysis assumes that the magnetospheric current transverse to the background magnetic field occurs principally because of the pressure gradients. It also assumes that FACs are generated when and where the transverse current diverges (or converges) to the ionosphere. A total of 97 crossings of the large-scale FACs by the DMSP F7 satellite in the dawn sector were analyzed to show that the locations of the ion pressure distributions are almost identical to those of the FACs. The distributions have peaks which are very close to the latitudes of the interface between region 1 and region 2 currents, and have the positive and the negative gradients in these regions, respectively. This demonstrates that the dawnside region 1 and 2 currents are derived from a common magnetospheric current, which is driven by pressure gradients. Very recently equatorial pressure maps were also obtained by Wing and Newell $(1998,2000)$ by computing ion pressure from auroral ion distribution data, for multiple low-altitude DMSP passes, allowing them to evaluate the contribution to Birkeland currents of the ion pressure during quiet times, of the order of $0.6 \mu \mathrm{A} / \mathrm{m}^{2}$.

Another, although complementary approach, has been developed by Galperin et al. (1992), and Galperin and Bosqued (1998). They assume that the generation of inverted-V FACs takes place around a narrow magnetic field minimum in the nightside near-Earth neutral sheet. It was suggested that the radial magnetic field profile in the inner plasma sheet, (or more precisely, in a region of dipole-to-stretched magnetic field transition close to the inner edge of the cross-tail current, which flows in the dawn-dusk direction), maximizes near its inner edge. This generates an additional magnetic field in the direction opposite to the dipole geomagnetic field, when the inner edge of the cross-tail current is quite sharp, and the cross-tail density decreases sufficiently with distance down the tail. The resulting radial magnetic field profile forms a narrow minimum at or close to the region of strong stochasticity for the plasma sheet ions where their bounce motion resonates with the Larmor rotation. As a consequence, the non-adiabatic westward motion of the ions leads to an additional westward line current in this direction. A FAC is created along the magnetic field minimum shell due to an azimuthal divergence of the cross-tail and westward line currents. A case study of auroral arc formation during a long time interval of stationary magnetospheric convection was analyzed using the AUREOL-3 satellite data (Galperin and Bosqued, 1999). The modelled FAC distribution agreed well with the measured characteristics of the FACs associated with the equatorial arc.

To summarize this brief introduction, the idea of an upward-directed FAC, generated by the pressure gradients is becoming more popular in spite of some difficulties related, among other things, to explaining the thermalization of the collisionless plasma in the plasma sheet.

This work is devoted to a statistical study of multiple inverted-Vs observed by the AUREOL-3 satellite. From the visual observation of electron spectrograms recorded over the two year mission (November 1981-December 1983) on more than 1000 auroral passes, it was evident that inverted-Vs are a rather common feature of the electron precipitation, first studied in detail by Bosqued et al. (1986). Among other properties it was shown that 
inverted-Vs extend over a latitudinal width of 50 $200 \mathrm{~km}$ at ionospheric altitudes. One of the most remarkable features evidenced but not studied then, was the presence on a given auroral pass of multiple inverted-V structures not randomly distributed but rather grouped in "families" of 2, 3, 4 or more successive (in space) structures. These "families" were suspected to result from some unknown stratification mechanism acting in the ionosphere or in the magnetosphere and which divided an initial and unique structure.

Observations of large-scale multiple inverted-V structures are not uncommon in the literature, as demonstrated, for instance, by the convincing examples presented in the eighties by Fennell et al. (1981), Klumpar et al. (1981) and Heelis et al. (1981) in the same AGU Monograph or, very recently, by McFadden et al. (1999). Series of parallel "arcs" having a typical thickness of $\sim 100 \mathrm{~km}$ and separated by $\sim 100 \mathrm{~km}$ were also observed in the dusk and pre-midnight sectors by the UV imager onboard the Freja satellite, accompanied by quasi-periodic electric fluctuations (Johnson et al., 1998). Often in these examples (Heelis et al., 1981) successive inverted-Vs occur simultaneously with successive changes in the gradient of the ionospheric electric field. This resulting electric field at ionospheric altitudes should reflect the distribution of the equatorial magnetospheric potential, but modified by the field-aligned potential drops accompanying each inverted-V. This idea was also put forward by Lyons $(1980,1981 \mathrm{a}, \mathrm{b})$ in his model of discrete precipitating structures, which necessary appear in the presence of large variations in the gradient of the magnetospheric potential.

In this work our intent is to explore the theory of the ionosphere-magnetosphere coupling developed by Tverskoy $(1982 \mathrm{a}, \mathrm{b})$ that seems to be the only one able to predict the large-scale stratification of the upward FAC. Tverskoy (1982a) and Antonova et al. (1998) have solved the fundamental equation of the magnetosphere-ionosphere coupling Eq. (2) assuming the relationship between potential and pressure variations is known. With a number of hypotheses (that will be questioned in this paper), they showed that potential variations (and FACs successive structures) obey a Schrödinger type equation with the number of zeros determined by a key parameter relating different properties of the ionospheric and plasma sheet plasmas. In this way the number of current sheets (and subsequently inverted-V structures) resulting from the upward FAC stratification can be in principle predicted.

As shown later, low-altitude polar-orbiting satellite measurements are able to provide all the values needed to determine the experimental magnitude of this parameter and to compare it with the predicted one. Therefore, an experimental study of the FAC stratification and of the formation of multiple inverted-V structures can be decisive for an indirect verification of the pressure gradient mechanism of FAC generation. The first attempt was made by Antonova et al. (1993, 1998), using the Intercosmos-Bulgaria (IB)-1300 satellite data. It was shown that the proposed mechanism gives a good description of the auroral zone-equatorial plane coupling. Nevertheless, the limited number of auroral oval crossings (less than 30 events available) as well as limitations of the IB-1300 instrumentation did not permit a good statistical analysis. Fortunately, the wider database of inverted-V events observed by the AUREOL-3 satellite will be used to perform a more accurate and detailed study than previously done with IB-1300.

This study is organized as follows: Sect. 2 contains a brief sketch of Tverskoy's stratification theory. Section 3 includes the AUREOL-3 instrumentation description and the methodology of data analysis followed to evaluate the central parameter of the theory. Event analysis presented in Sect. 4 illustrates the main features of the multiple inverted-V structure formation. Finally, Sect. 5 contains the results of the statistical study, while Sect. 6 provides a summary and conclusions.

\section{Tverskoy's stratification theory}

As mentioned before, the stratification theory proposed by Tverskoy (1982a, b, 1983) is based on the assumption that there is magnetostatic equilibrium during the stratification of plasma into separate structures. This coincides with the observed pressure isotropy in the centre of the plasma sheet (Christon et al., 1991; Huang and Frank, 1994). The fundamental equation of the magnetosphere-ionosphere coupling Eq. (2) can be solved only when the relationship between potential and pressure variation is known. Tverskoy (1982a) and Antonova et al. (1998) have solved this problem for the case in which the spatial scale of electric field variations is comparable with the Larmor radius of ions. In the simplest case the pressure variation is proportional to the variation of the magnetospheric potential, and it is important to note that it is not relevant whether the large-scale undisturbed potential and plasma pressure are related by the same law.

Thus as zero and first approximation, Eq. (2) can be written as:

$$
\begin{aligned}
& \operatorname{div}\left(\Sigma_{0} \vec{\nabla} U_{0 i}\right)=j_{0} \quad \text { and } \\
& \operatorname{div}\left(\Sigma_{0} \vec{\nabla} u_{i}+\Sigma^{\prime} \vec{\nabla} U_{0 i}\right)=-j_{0} \frac{e u_{m}}{T_{i}}
\end{aligned}
$$

respectively. Here $U_{0 i}, \Sigma_{0}$, and $j_{0}$ are the undisturbed values of ionospheric potential, ionospheric conductivity (including conductivity from solar radiation as well as from undisturbed electron flux), and field-aligned current, respectively, $u_{i}, u_{m}$ are the ionospheric and magnetospheric potential disturbances, and $\Sigma^{\prime}$ is the ionospheric conductivity disturbance resulting from variations of energy and energy flux of precipitating electrons.

The solution of Eq. (4) can be unstable if the potential variation leads to FAC increases both in the ionosphere and in the magnetosphere. As shown in Antonova et al. (1998), a specific instability develops 
only in the case of the upward FAC, leading to their stratification.

For constant conductivity, Eq. (4) simplifies as

$\Delta u_{i}+\frac{e j_{0}}{\Sigma_{P} T_{i}} u_{m}=0$

where $\Sigma_{P}$ is the height-integrated Pedersen conductivity, and $T_{i}$ is the ion temperature (in the plasma sheet).

When the current stratifies, forming azimuth-extended current sheets, and the field-aligned potential drop is small $\left(u_{i}=u_{m}=u\right.$ i.e. the same disturbance in the magnetosphere and the ionosphere), Eq. (5) can be written as

$\frac{\mathrm{d}^{2} u}{\mathrm{~d} \xi^{2}}+\Gamma(\xi) u=0$

where $\xi=x / L$ is the dimensionless distance along the meridional direction, and $L$ is the half-latitudinal width of the upward current band. In this way it is possible to introduce a dimensionless parameter

$\Gamma(\xi)=\frac{e j_{0}(\xi) L^{2}}{\Sigma_{P} T_{i}}$

where $e$ is the electron charge.

As seen, Eq. (6) has a Schrödinger equation form only in the case of the upward field-aligned current $\left(j_{0}(\xi)>0\right)$. In this case the number of zeros $(m)$ is mainly determined by the depth of potential well: $\Gamma=e j^{\max }$ $L^{2} /\left(\Sigma_{P} T_{i}\right)$, where $j^{\max }$ is the maximum value of $j_{0}(\xi)$ and the number of structures formed $N=m / 2+1$. Tverskoy (1982b) has shown that for a FAC density $j_{0}(\xi)=j^{\max } \cosh ^{-2}(\xi), \Gamma=1$ corresponds to unstratified current, $\Gamma=6$ relates to the formation of double sheet current structure; if $\Gamma$ is in the range of 20 to 40 , an unstratified current will separate into three sheets. These values may vary weakly depending on the shape of the unstratified current; however the number of current sheets should increase as square root of $\Gamma$.

It is necessary to mention that $\Gamma$ is insensitive to the choice of the altitude in which the current density and the structure's half-width are determined. This results from the fact that the magnetic flux is conserved along the magnetic field line. Therefore the current density decreases and the $L^{2}$ increases with altitude, compensating each other.

Antonova and Stepanova (1988) and Antonova et al. (1988) have studied the influence of the presence of the field-aligned potential drop and the variation of the ionospheric conductivity on the stratification. It was shown that these effects could increase or decrease the $\Gamma$ value and lead to stratification asymmetry. Here we will study the large-scale stratification only. In this respect it is possible to neglect the effects mentioned and consider only the relation (7).

\section{Instrumentation and methodology}

Our analysis is based on experimental observations of the large-scale multiple inverted- $\mathrm{V}$ electron precipitation made by the AUREOL-3 (A3) spacecraft between
November 1981 and December 1982. The three-axis stabilized AUREOL-3 satellite was launched on September 21, 1981 into a low-altitude polar orbit (perigee: $410 \mathrm{~km}$, apogee: $2000 \mathrm{~km}$, inclination: 82.5) and was in operation for nearly six years. Particle data with a time resolution of 1.6-3.6 s. were provided by the SPECTRO instruments which included a set of multidirectional electrostatic spectrometers (ROBE instrument) scanning the $\sim 0.02-22 \mathrm{keV}$ energy range, and viewing different pitch angle directions, and two energy-mass spectrometers (ION1 and ION2) separating $\mathrm{H}^{+}, \mathrm{He}^{+}$and $\mathrm{O}^{+}$ ion species from several bands between $\sim 10 \mathrm{eV} / \mathrm{e}$ and $14 \mathrm{keV} / \mathrm{e}$, along 2 directions, $\approx 60^{\circ}$ and $\approx 120^{\circ}$ pitch angle, (Bosqued et al., 1982). Magnetic field three-component measurements were made by the TRAC instrument (Berthelier et al., 1982) with a time resolution up to $80 \mathrm{~ms}$.

More than 1000 passes of the A3 satellite have been examined in order to select inverted-V events according to the following steps: (a) visual identification from the archived electron spectrograms and search for the characteristic energy-time (latitude) variation of inverted-Vs, then, (b) check for existence of three consecutive spectra with $E_{\text {peak }} \geq 1 \mathrm{keV}$ in a given "inverted-V" structure. Moreover, to restrict our analysis to the clearer cases, three additional scientific criteria were added: (1) elimination of cases when the A3 pass was not meridian (within $\sim 30^{\circ}$ ), (2) elimination of inverted-Vs observed on the dayside $(06-18 \mathrm{H})$ and/or on the polar cap, and (3) elimination of inverted-Vs located near the boundary of the polar cap (presumably mapping in the distant tail).

According to these criteria the minimum width of retained structures is in the order of $3 \times 1.6 \mathrm{~s} \times 8 \mathrm{~km} / \mathrm{s}$ $\sim 40-50 \mathrm{~km}$ at ionospheric altitudes. It may be noted that elimination of passes when A3 moves tangentially to the auroral oval, spending longer time crossing inverted-Vs, precludes a more detailed study of thinner structures. However this study was included in a previous work (Bosqued et al., 1986).

The final database contains 154 passes with at least one inverted- $\mathrm{V}$ and is almost identical to the one used by Bosqued et al. (1986), with the addition of some extra events not yet processed at the time of this publication. The majority of events is detected during quiet and moderately disturbed periods: as shown in Fig. $166.2 \%$ of inverted-Vs occur for an instantaneous AE index less than $300 \mathrm{nT}$, confirming that inverted-Vs may occur independently of the magnetospheric (auroral) activity but are more probably a significant signature of the substorm onset and growth phase (Galperin, 1994; Galperin and Bosqued, 1999). This occurrence of multiple structures also seems independent of any specific level of auroral activity.

The methodology followed was originally developed for the analysis of the Intercosmos-Bulgaria-1300 (IB1300) satellite data (Antonova et al., 1991, 1998) and has been easily adapted to the larger A3 database. First, particle data are used to determine the upward current density, the half-width of the current band, the heightintegrated Pedersen conductivity and the ion tempera- 


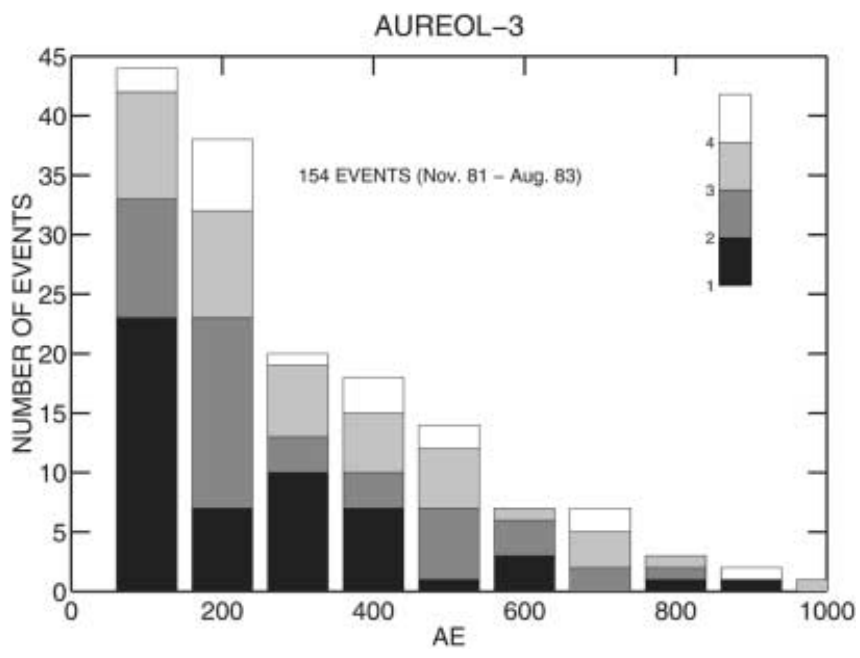

Fig. 1. Distribution of the 154 events versus the instantaneous value of the AE index, split up into four groups $(1,2,3, \geq 4)$ according to the number of inverted-Vs observed in each

ture of the plasma sheet. When magnetic field data are available, they are used to confirm the latitudinal extent of the upward directed current band. These experimental values are then inserted in Eq. (7) to calculate an experimental $\Gamma$ parameter and compare it with the theoretical value corresponding to the number of observed current sheets (inverted-V structures):

$\Gamma^{\exp }=10 \frac{j^{\max } L^{2}}{\left\langle\Sigma_{P}\right\rangle\left\langle T_{i}\right\rangle}$

where $j^{\max }$ is the maximum value of the undisturbed FAC density expressed in $\mu \mathrm{A} \mathrm{m}^{-2}, L$ is the half-width of upward field-aligned current band in $10^{5} \mathrm{~m},\left\langle\Sigma_{P}\right\rangle$ is the mean height-integrated Pedersen conductivity in the ionosphere in $\mathrm{S}$, and $\left\langle T_{i}\right\rangle$ is the mean temperature of magnetospheric ions in $\mathrm{keV}$. The coefficient 10 results from unit conversion, from SI to more traditional space physics units.

Energy electron distributions within inverted-Vs generally display different components (Chiu and Schultz, 1981): (a) "primary" electrons directly injected from the magnetosphere (plasma sheet), then accelerated into the loss cone to energies higher than $E_{\text {peak }}=\mathrm{eV} \|$ (V\|: parallel potential drop) and, superimposed on this, (b) "secondary" ionospheric electrons with energies lower than $E_{\text {peak. }}$. Evans (1974) and Pulliam et al. (1981) have proposed a model which accurately describes electron spectra (including A3 spectra) commonly observed in inverted-Vs, by demonstrating that secondary electrons are backscattering ionospheric electrons bouncing up and down between their atmospheric mirror point (below the satellite) and the repelling potential drop (above the satellite). The calculation of the current density requires knowledge of the full distribution function; unfortunately only the $20-90^{\circ}$ pitch angle range was scanned by A3 and the distribution of precipitating electrons is assumed to be practically flat. Partial information on the upgoing electron flux in the 100-10 $000 \mathrm{eV}$ range is given by an extra sensor (TBE2) and helps to evaluate the upward fluxes in this energy range. As demonstrated by Sugiura et al. (1984) and Hoffman et al. (1985) using DE1/2 satellites, the upward directed current is mainly carried by hot accelerated electrons. Also, in the present work, we adopt the same integration region as in Bosqued et al. (1986): the upward directed electron current density is simply obtained by integrating the primary (magnetospheric) electron flux in the loss cone, from $0.8 * E_{\text {peak }}$ to $\sim 22 \mathrm{keV}$ only.

The height-integrated Pedersen conductivity is computed as a function of the incident energy flux measured at the A3 altitude (obtained by integrating the differential flux over the loss cone) and/or the average energy, using the empirical formulas proposed by Harel et al. (1981) and Spiro et al. (1982). UV and ion contributions are neglected for this study in the nighttime sector. These methods are similar to these subsequently proposed by Robinson and Vondrak (1984), Robinson et al. (1987), and Hardy et al. (1987), (see (Ahn et al., 1998 for a review) but oversimplified compared to more recent models (Gjerloev and Hoffman, 2000). Nevertheless, all methods predict that the conductivity increases at the same rate as the square root of the precipitating electron energy flux, and calculated height-integrated conductivities differ by less than 10-15\%, which is much less than the variation of the conductivity across the auroral oval.

The half latitudinal width of the upward FAC band was found by correcting for the angle between the A-3 trajectory and the corrected geomagnetic meridian, which was less than $\sim 30^{\circ}$ for all selected cases. In that case we implicitly assume that all inverted-Vs are eastwest aligned. Magnetometer data, available only for a minority of events, confirms this hypothesis.

The magnetospheric ion temperature is determined by using a Maxwellian distribution function to fit the hot ion distribution (between $\sim 1$ and $15 \mathrm{keV}$ ) measured by the most sensitive sensor of the ROBE instrument. When the ion count rate is too low, four successive spectra are accumulated to extract a significant value for $T_{i}$. Moreover, in a few examples, results of the ION1 sensor (energy per mass analysis, pitch angle $\sim 60^{\circ}$ ) are used to compute a new $T_{i}$ value that generally agrees well with the previous one.

Throughout this study we will make the following assumptions: (1) to evaluate the maximum value $j^{\max }$ of the undisturbed FAC, according to the approximate profile introduced in Antonova et al. (1998), we assume conservation of the total current and the total width of the current band, (2) the variations in the ion temperature and in the Pedersen conductivity are small enough so that their mean values can be used, and (3) the ionospheric current flows mainly across the auroral oval. The last assumption allows the problem to be treated as one-dimensional. Antonova et al. (1991, 1998) have shown that these three conditions are generally fulfilled during the steady state. Validity of these assumptions will be discussed in Sect. 6 .

In computing the $\Gamma$ parameter most errors occur in calculating the ionospheric conductivity and tempera- 
ture, about $10-20$ and $20-30 \%$, respectively. This produces a final error for $\Gamma$ of about $50 \%$, which is quite high. However, it must be kept in mind that the $\Gamma$ values associated with the formation of one, two or three current sheets differ significantly from each other. Therefore, although the study of an individual event may not be convincing, considering the high level of relative error, the statistical study of large quantities of data could reveal whether there is a systematic trend in the behaviour of the $\Gamma$ parameter.

\section{Case study}

The main objective of this section is to illustrate the application of elaborated methodology on two examples of multiple inverted-V events. Two consecutive and typical passes of the A3 satellite in the midnight side of the auroral oval (Northern Hemisphere) were selected (December 13, 1982). Figure 2 displays $\sim 5$ min of data showing a first meridional crossing (Orbit 5914, $23.9 \mathrm{H}$ MLT) of three large-scale inverted-V structures between 20:23:20 and 20:24:45 UT (electron spectrogram, second panel from top), the poleward structure centred on $\sim 69.8^{\circ}$ invariant latitude (ILAT) and the equatorward one on $\sim 66.4^{\circ}$. Field-aligned current profiles obtained from magnetic field variations (using Maxwell equations) and electron spectra integration are plotted in the first and third panels (from top), respectively. Both profiles confirm the stratification into three sheets. Maximum local density reaches $5 \mu \mathrm{A} \mathrm{m}^{-2}$ for the first event for the FAC carried by electrons and only $\sim 2 \mu \mathrm{A} \mathrm{m}^{-2}$ for the FAC deduced from magnetic field variations. Variations are similar for the three main structures although for this pass the FAC carried by primary electrons is systematically higher by a factor of 2. Such differences were already noted by Sugiura et al. (1984) and, in the case of A3 measurements, can be due either to difficulties in extracting magnetic field variations from a noisy magnetic field signal, or to geomet-
AUREOL-3 1982-12-13 ORBIT:5914-5 MODE:511 SEQ:3192

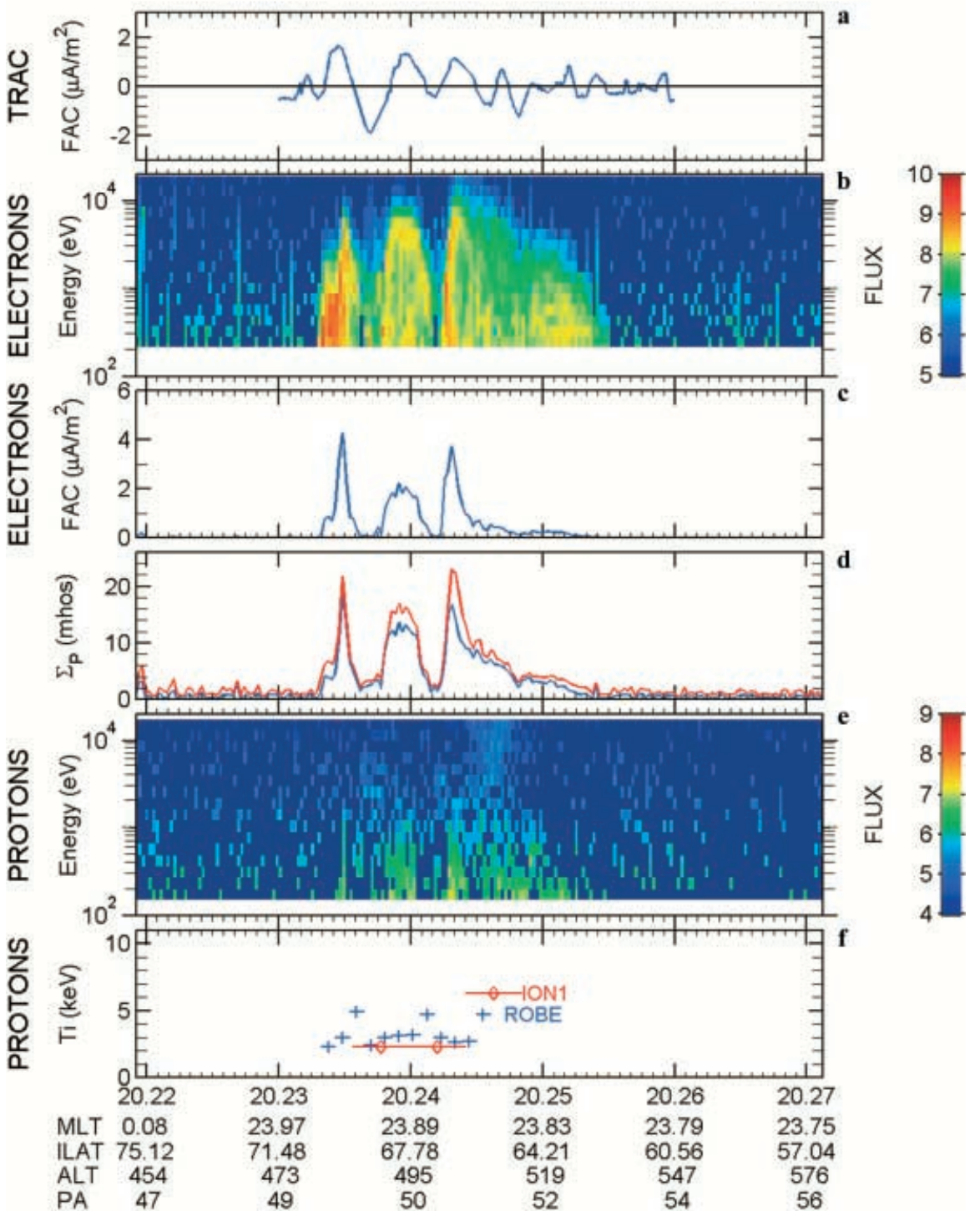

Fig. 2. Example of multiple (three) inverted-V structures observed by the AUREOL3 satellite in the midnight sector during a northern pass (pass 5914) on December 13, 1982, around 2020 UT. From top to bottom: a Field-aligned current deduced from magnetic variations $\left(\mu \mathrm{A} / \mathrm{m}^{2}\right)$, b electron flux spectrogram, $\mathbf{c}$ field-aligned current carried by hot electrons $\left(\mu \mathrm{A} / \mathrm{m}^{2}\right)$, d Pedersen conductivities (S) according to Harel et al. (1981) and Spiro et al. (1982) expressions (red and blue lines, respectively), e proton flux spectrogram, $\mathbf{f}$ ion temperature $(\mathrm{keV})$ provided by the ROBE and ION1 instruments 
rical factors of the electron sensors not being perfectly adjusted. Nevertheless, the currents obtained are of the right order of magnitude when structures of a $\sim 100 \mathrm{~km}$ scale are considered (Stasiewicz and Potemra, 1998).

The maximum value of the non-stratified FAC is calculated to be $1.4 \mu \mathrm{A} \mathrm{m}^{-2}$ following the methodology developed by Antonova et al. (1998). The heightintegrated Pedersen conductivity of the ionosphere (third panel from bottom), calculated according to Harel et al. (1981) and Spiro et al. (1982) relations, are similar and appear highly structured in phase with the FAC density, and have a mean value of $6.9 \mathrm{~S}$. In all the studied examples the contribution of electrons $<1 \mathrm{keV}$ to $\Sigma_{P}$ is not significant. The values obtained, $10-20 \mathrm{~S}$, are coherent with DE2 average conductivities measured in the boundary plasma sheet (BPS) region (Gjerloev and Hoffman, 2000).

The trajectory of the A3 satellite coincides with the geomagnetic meridian, and the half-width of the FAC band is $\sim 3.73 \times 10^{5} \mathrm{~m}$ (at the spacecraft altitude, $\sim 500 \mathrm{~km}$ ). Finally, the ion temperature (see bottom panel) appears highly variable for this pass, with an average value of $3.4( \pm 1.8) \mathrm{keV}$. As shown by the ion spectrogram (second panel from bottom), the ion count rate is exceptionally low for this pass and integration over four consecutive ion spectra was necessary to obtained reliable values for $T_{i}$. Values of $T_{i}$ as deduced from the $\mathrm{H}^{+}$energy spectra provided by the ION1 instrument are also given for comparison, although only 4-point spectra were recorded at that period of the A3 mission.

Inserting $j^{\max }=3,\left\langle\Sigma_{P}\right\rangle=6.9,\left\langle T_{i}\right\rangle=3.4, \quad L=3.73$ into Eq. (6) we obtain $\Gamma \sim 7.5$, which differs significantly from the predicted value of 20 for three structures. However, it is important to note, that this theoretical $\Gamma$ value is obtained using a number of hypotheses which determine the choice of the nonstratified current profile and assume a constant heightintegrated Pedersen conductivity. These points will be discussed at the end of this work.

During the next pass (pass 5915, December 13, 1982, $\sim 22: 12$ UT) we again observe three large-scale invertedV structures in the electron spectrogram (Fig. 3), still in the midnight sector $(\sim 00 \mathrm{H} 20 \mathrm{MLT})$ and between $\sim 71.6$ and $\sim 67.5$ ILAT. However the total electron flux within the poleward structure located at $\sim 71.5$ ILAT, and

AUREOL-3 1982-12-13 ORBIT:5915-5 MODE:511 SEQ:3197

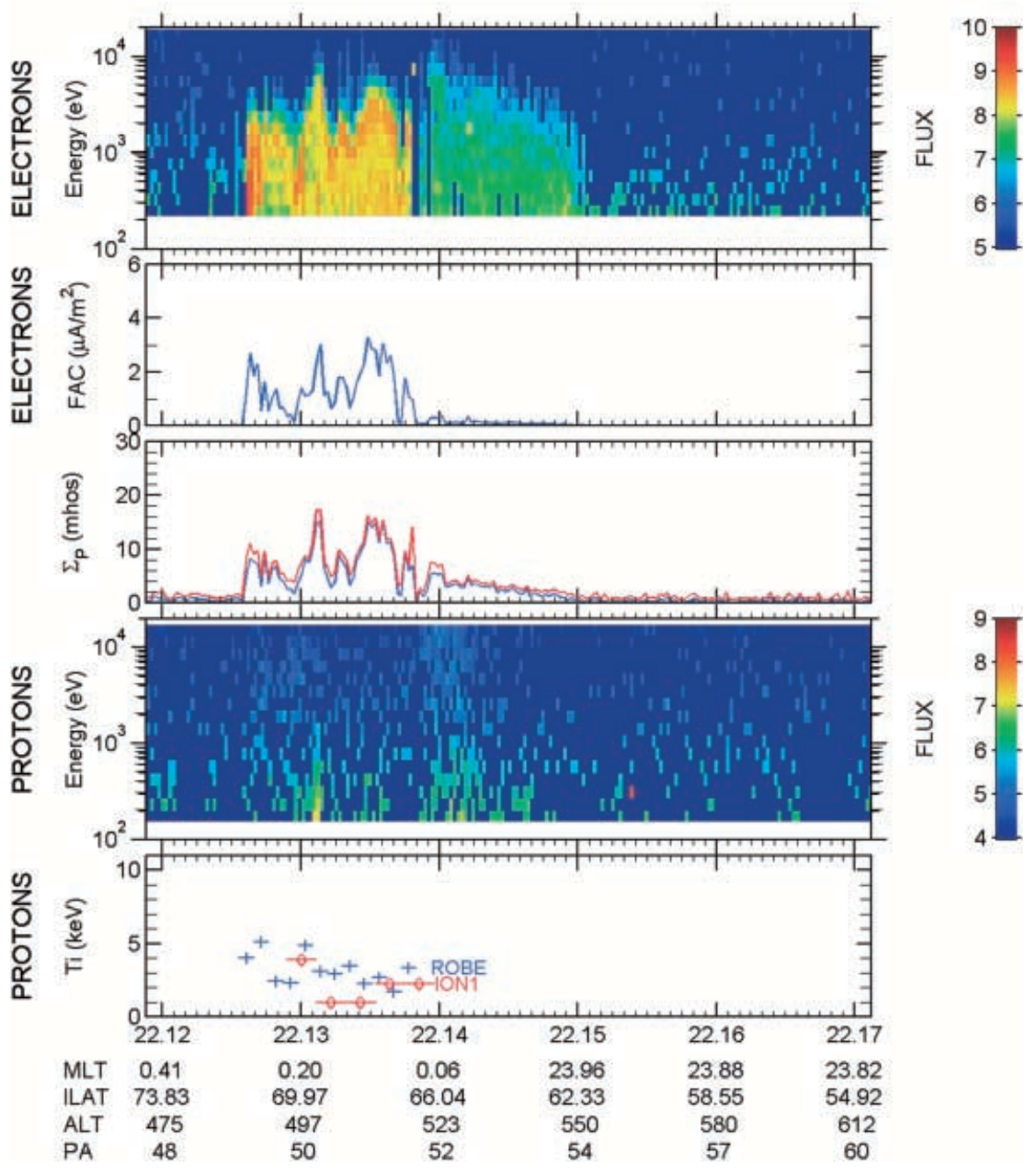

Fig. 3. Next pass (pass 5915) around 2212 UT, the same day. Format identical to Fig. 2, except that the field-aligned current deduced from magnetic variations was not available for this pass 
therefore the FAC current, is decreasing. The corresponding values of the non-stratified FAC, heightintegrated conductivity, and half-width of the current band are $2.0 \mu \mathrm{A} \mathrm{m}^{-2}, 7.5 \mathrm{~S}$, and $2.67 \times 10^{5} \mathrm{~m}$, respectively. The average value of the ion temperature in this case is approximately $3.1( \pm 0.6) \mathrm{keV}$, producing the experimental value of $\Gamma \sim 6.1$ for this "family" of events. It can be noted that new substructures at smaller scales could be easily recognized within the three largescale inverted-V structures (see for example the sharp maxima in the FAC profile near 2212:48 and 2213:17 UT). This example introduces the difficulty often met in determining the number of observed structures: larger structures are often divided into smaller scale current sheets with an appreciable current.

Both the interplanetary magnetic field (IMF) measured by the ISEE-3 during December 13-14, 1982, and the Dst index variation for this period showed that interplanetary conditions and the general state of the magnetosphere during that period were very quiet. The auroral electrojet indices plotted in Fig. 4 also indicate that the geomagnetic activity was low, although two small geomagnetic substorms occurred between 20:40 and 21:50 UT, having a maximum AE-index of about $500 \mathrm{nT}$. The first series of inverted-Vs (orbit 5914) was observed during the growth phase of the substorm and the second one (next orbit, orbit 5915) was crossed at the end (vertical lines). Therefore, we can suppose that during the two consecutive crossings of the auroral oval, $\sim 110$ minutes apart, we observed the same large-scale and stable auroral context with a similar three-current sheet structure roughly shifted poleward but with more irregular structuring during the second pass. We do not know whether inverted-Vs can remain stable for periods exceeding 20-30 min (Lin et al., 1985; Thieman and Hoffman, 1985). However of 19 cases of inverted-V observations in consecutive orbits, exactly the same number of structures was observed in eight cases. In
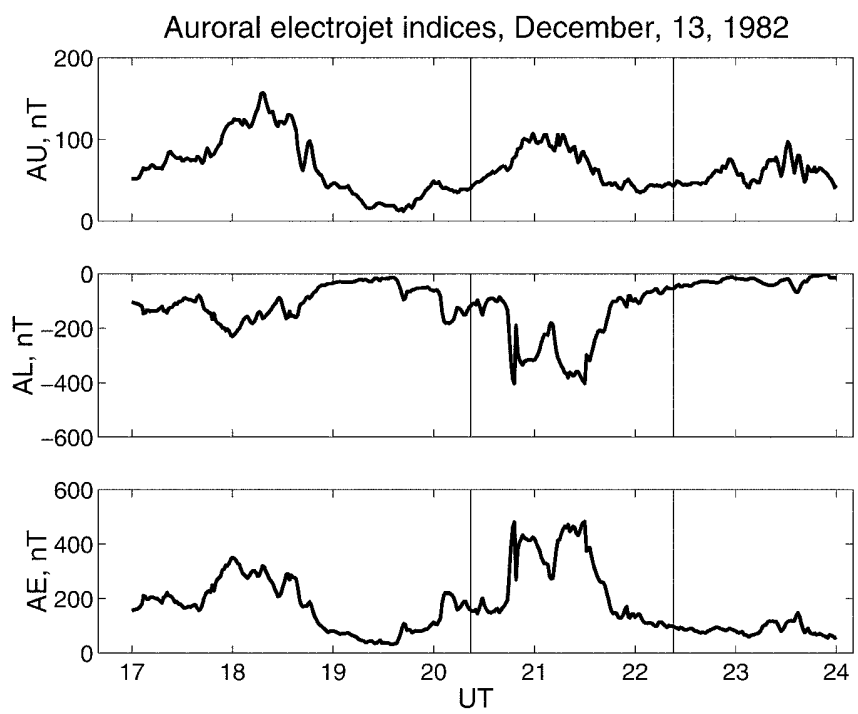

Fig. 4. One minute auroral electrojet indices, December 13, 1982. Vertical lines indicate the time of the A3 passes displayed in Figs. 1 and 2 nine cases the number of structures differed by one, and in only two cases was the observed picture completely different. This testifies in favour of the possibility of such stability for periods of about $2 \mathrm{~h}$. (the time between two consecutive passes), when magnetospheric conditions near the equatorial plane do not change significantly during the corresponding time interval.

\section{Statistical results}

We now present the results of the statistical study carried on the 154 single or multiple inverted-V events observed by the A 3 satellite. Figure 5 gives an overview of the basic distribution of obtained $\Gamma$ values according to the MLT sector of observation, subdivided into different classes corresponding to the formation of one, two, three, etc., structures. As noted, inverted-Vs were randomly selected in the $18 \mathrm{H}-06 \mathrm{H}$ sector, and distribution of these points according MLT does not represent their real frequency of occurrence. Nevertheless it does reflect that the frequency of occurrence is higher in the 20-01H MLT sector (Bosqued et al., 1986). The most significant result is that, within every MLT sector, the $\Gamma$ value associated with each event (i.e. with each "family" of single or multiple inverted-Vs) increases with the observed number of current sheets. This trend is predicted by Tverskoy theory developed in Sect. 2 . The number of observed events naturally decreases with the increase in the number of individual inverted-V structures observed in each event: one-sheet structures (53 events) were characterized by $\Gamma=0.9 \pm 0.6$, the average values of $\Gamma$ for two (44 events), three (40 events), four (12 events), five (three events), and six (two events) sheet structures were $\Gamma=4.5 \pm 3.8, \Gamma=12.4 \pm 14.2$, $\Gamma=13.7 \pm 6.6, \Gamma=6.3 \pm 3.5$ and $\Gamma=31.3 \pm 21.6$, respectively. As a general trend, it seems that the $\Gamma$ values are slightly higher around 20-22 MLT. Figure 6 synthesizes the variation of average $\Gamma$ values (and standard

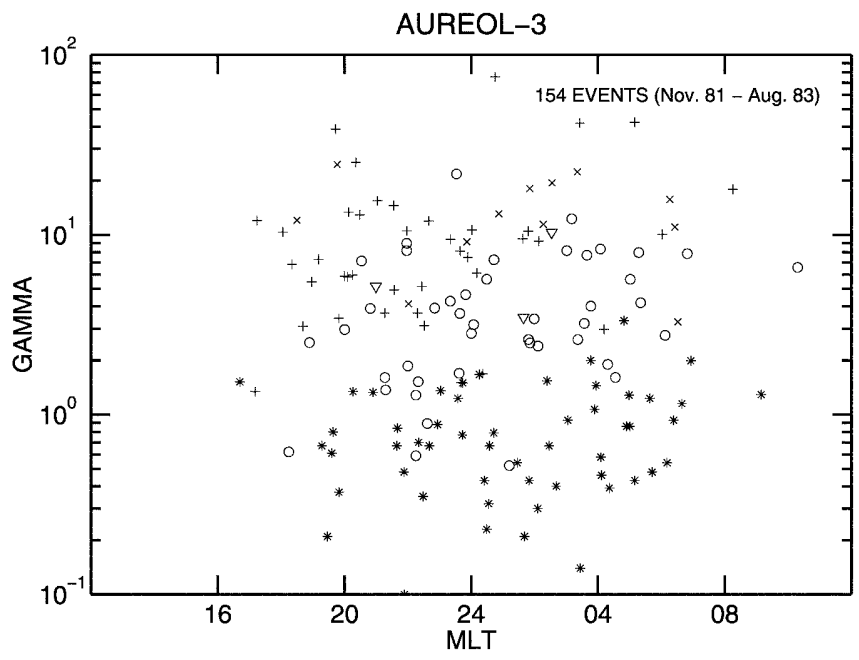

Fig. 5. Values of $\Gamma$ for each event as a function of MLT, according to the number of detected sheets/inverted-Vs: one $(*)$, two (o), three $(+)$, four $(\times)$, five $(\nabla)$, and six $(\diamond)$ 
deviations) with the number of current sheets detected by the A3 satellite (asterisks). The systematic growth of the average values of $\Gamma$ with the number $N$ of current sheets, at least for $N \leq 3$, is well evidenced and follows a fitting law $\log _{10} \Gamma=2.41 \cdot \log _{10} N-0.065$ (black fitting line), i. e. in fact $\Gamma \propto N^{2}$ This variation agrees with the theoretical predictions of the Tverskoy theory summarized in Sect. 2 and plotted on the same diagram (diamonds), and coincides rather well with previous results obtained by Antonova et al. (1998) also plotted for comparison in Fig. 6 (symbol). However, as seen, the average $\Gamma$ values obtained using IB-1300 data are slightly higher than those obtained in the present work using A3 data. This small and systematic shift may be related to the difficulties in the accurate determination of the ion temperature using Intercosmos-Bulgaria-1300 data. The poor knowledge of detectors installed onboard IB-1300, calibration uncertainties, electron overflow in ion detectors when the electron flux was high, UV contamination and in-flight detector fatigue can account for temperature discrepancies and, to a lesser extent, of flux and current differences. However, in spite of these uncertainties, in both cases the $\Gamma$ value increases simultaneously with the number of structures formed and the agreement with the predictions is surprisingly excellent.

This interesting variation of the $\Gamma$ parameter being demonstrated, we searched for the physical parameter(s) controlling the $\Gamma$ value associated to each single or multiple inverted-V structure, $j^{\max }, \Sigma_{P}$ and $T_{i}$. As changes in the maximum (non-stratified) electron current and in the $\Sigma_{P}$ conductivity should be correlated, we have plotted in Fig. 7 the changes in $\Gamma$ as a function of $j^{\max }$ for each class of inverted-V structures. It can be noted that the $\Gamma$ values for each class remain relatively

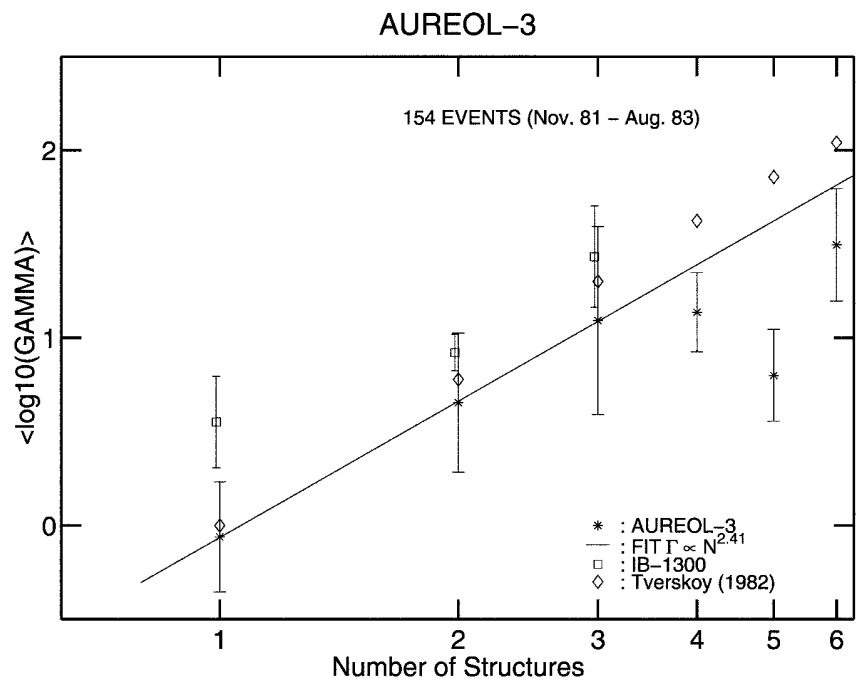

Fig. 6. Average value $(*)$ and standard deviation of the $\Gamma$ parameter as a function of the number $N$ of observed current sheets detected by the AUREOL-3 (A3) satellite. Results obtained using the Intercosmos-Bulgaria-1300 satellite data ( $\square$ ) (Antonova et al., 1998) and Tverskoy's predictions $(\diamond)$ are also plotted for comparison. The black line corresponds to the best fitting of the $\mathrm{A} 3$ data by the function $\Gamma \propto a N^{b}$ where $b \sim 2.41$

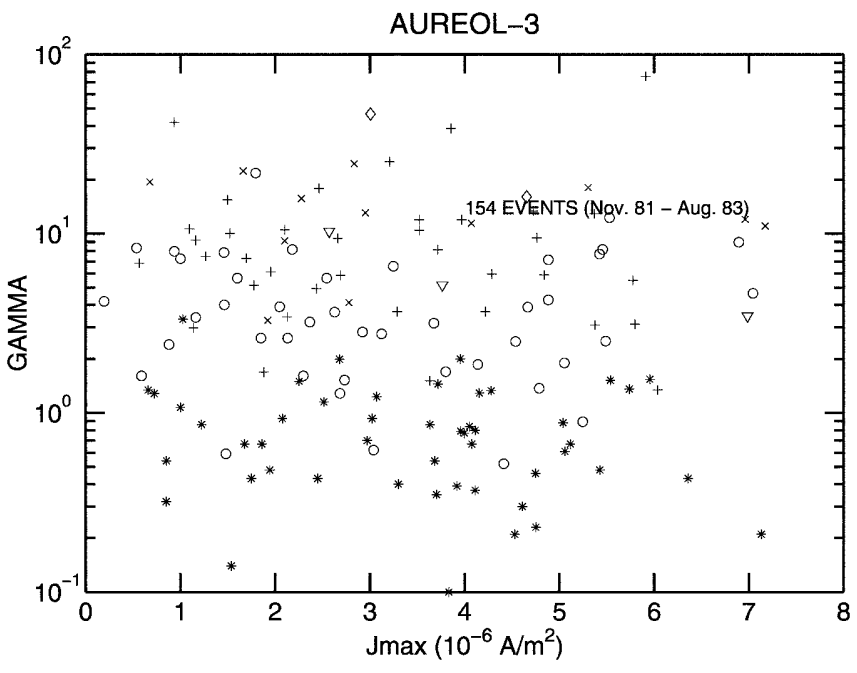

Fig. 7. Values of $\Gamma$ for each structure as a function of the maximum value of the non-stratified current, in $\mu \mathrm{A} \mathrm{m}^{-2}$. The symbols used to identify the number of inverted- $V$ current sheets are the same as in Fig. 5

unchanged for a large range of current densities extending up to $\sim 10^{-5} \mathrm{~A} / \mathrm{m}^{2}$. Although this conclusion must be tempered as the exact shape of the non-stratified current is largely unknown, $\Gamma$ seems practically independent of $j^{\max }$ (and of $\Sigma_{P}$ not shown). However, events with $\mathrm{n}>3$ structures seem to occur mostly when $j^{\max } \geq 3 \mu \mathrm{A} / \mathrm{m}^{2}$; this point will be discussed in the last part of this work, together with the "saturation" of $\Gamma$ observed for the same $n>3$ events (see Fig. 6).

To summarize, the $\Gamma$ parameter appears relatively insensitive to any of the parameters controlling its value. To confirm this, we may introduce the half "wavelength" $l$ of oscillations described by the differential Eq. $(5,6)$ :

$l=\left[\frac{\Sigma_{P} T_{i}}{e j^{\max }}\right]^{1 / 2}$

$l$ is related to $\Gamma$ through $l=\left(L^{2} / \Gamma\right)^{1 / 2}$ where $L$ is the half-latitudinal width of the upward current band.

Figure 8 shows the distribution of the latitudinal width $2 l$, obtained for each event according to Eq. (9), again, grouped according to the number of individual current sheets (identifications is the same as in Fig. 5). The most important result appears here, i.e. the average latitudinal width of each auroral "arc" stays remarkably constant, regardless of the total width of the full current sheet and the number of sub-structures included within this sheet. Normalized at ionospheric altitudes $(\sim 500 \mathrm{~km}), 2 l$ is approximately $180 \mathrm{~km}(\sigma=40 \mathrm{~km})$. This is close to the "wavelength" obtained directly as $2 * L / N(\sim 230 \pm 120 \mathrm{~km})$. This value is slightly higher than that obtained by Bosqued et al. (1986) for the inverted-V width, because it includes not only the width but also the distance between the two consecutive structures forming the same "family", although this distance was generally smaller than the structure width. Possible variations of $2 l$ with the MLT sector are displayed in Fig. 9 for each group of events, and 


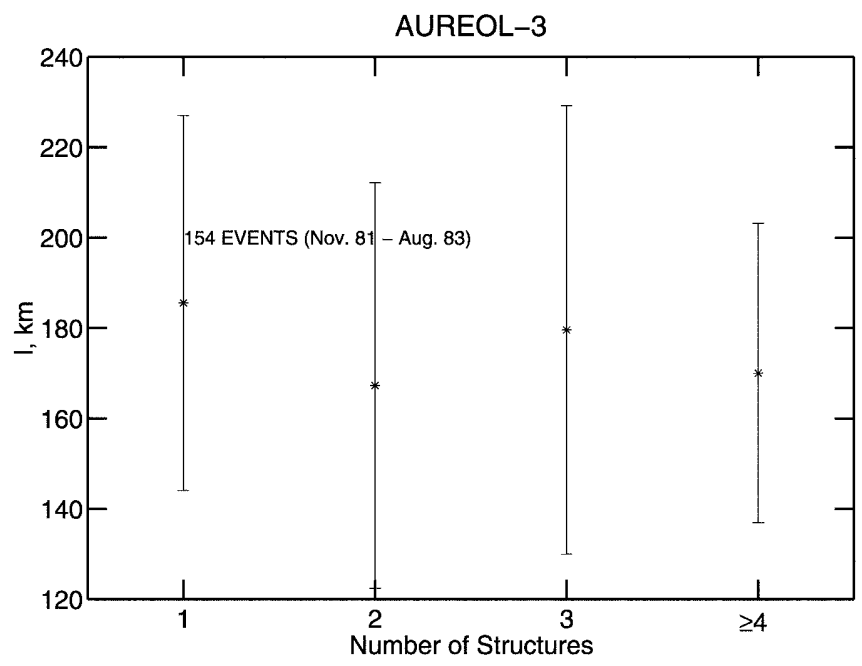

Fig. 8. Average value and standard deviation of the characteristic latitudinal width $2 l(\mathrm{~km})$ of the individual inverted-Vs, given by Eq. (9) as a function of the number $N$ of structures in each event

demonstrate that this width remains independent of the sector of observation. However, within the large dispersion of the observations, it appears that single and double events are wider in latitude around the premidnight sector, confirming the trend obtained by direct measurement of the inverted- $\mathrm{V}$ width noted previously in Bosqued et al. (1986).

\section{Discussion and conclusions}

We have studied 154 inverted-V events observed at low altitude, principally in the nightside auroral zone, by the AUREOL-3 satellite, and particularly the presence of multiple inverted-V structures detected during the same auroral crossing. At times, during quiet geomagnetic

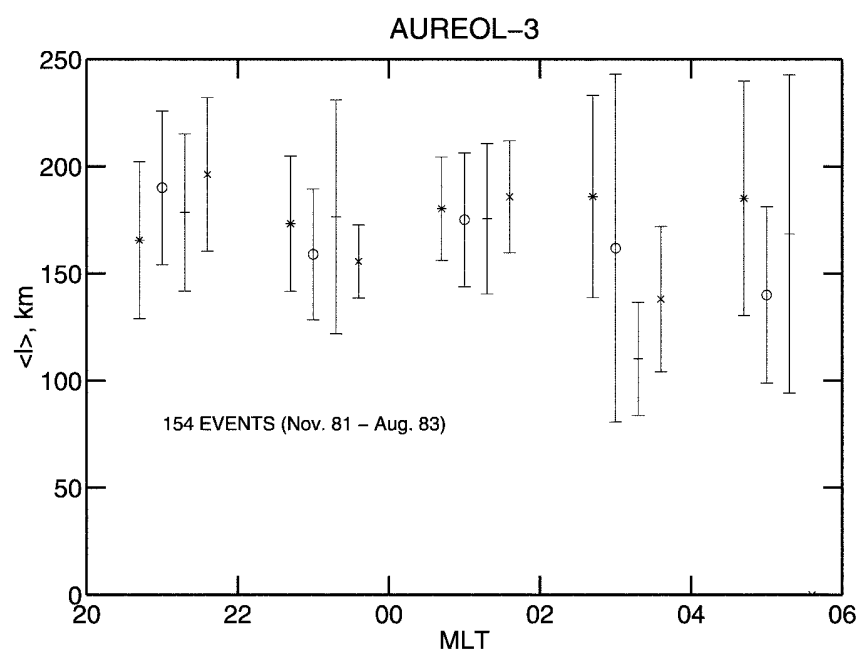

Fig. 9. Average value and standard deviation of the characteristic latitudinal width $2 l(\mathrm{~km})$ according to the MLT sector of observation; the set of events is split up into four groups $(1,2,3, \geq 4)$ according to the number of inverted-Vs observed in each conditions, similar latitudinal distributions of multiple structures were encountered on two consecutive passes in the auroral oval, demonstrating a significant lifetime of these multiple inverted-V structures. We tried to answer the basic question: are these structures randomly distributed in latitude or are they related to each other and result from an ionospheric and/or magnetospheric mechanism of stratification? For each event we have accurately determined the plasma parameters describing the inverted-Vs properties and performed a statistical study which provides a few reliable statistical trends. We have tried to understand their large-scale stratification within the framework of the Grad-Vasyliunas-BoströmTverskoy concept for the generation of field-aligned currents. The current generation is attributed to the existence of the plasma pressure gradients in the equatorial magnetosphere. Following this approach, Tverskoy's mechanism of the upward field-aligned current stratification predicts some periodicity in the potential profile (and therefore in the FAC profiles) that could account for oscillations experimentally observed in the auroral zone, such as successive inverted-Vs.

Our results can be summarized as follows:

1. It appears that the central parameter $\Gamma^{\exp }=10 \frac{j^{\max } L^{2}}{\langle\Sigma\rangle\left\langle T_{i}\right\rangle}$ proposed in Tverskoy, theory correctly quantifies the number of the individual and contiguous electron inverted-V structures observed on a given satellite pass.

2. The $\Gamma$ parameter appears as relatively robust and remains relatively stable regardless of the observed maximum current density and the related Pedersen ionospheric conductivity.

3 . The characteristic "wavelength" $\sim 230 \mathrm{~km}$ at ionospheric $(500 \mathrm{~km})$ altitudes (the inverted-V width and the distance between two consecutive structures of the same "family") stays remarkably constant even in the case of parallel multiple inverted-Vs.

This last result, which concerns the scaling of inverted- $\mathrm{V}$ structures as observed in the auroral regions, is of crucial importance. The periodicity in the potential profile (and subsequently in the FAC profiles) predicted by the Tverskoy theory could be manifested by oscillations in the field-aligned currents experimentally observed in the auroral zone, for instance as successive inverted-Vs. The wavelength or characteristic width, $l=\left[\frac{\Sigma_{p} T_{i}}{e j^{m a x}}\right]^{1 / 2}$, in order of $\sim 180 \mathrm{~km}$, directly expressed in terms of plasma sheet properties, corresponds remarkably well to the average experimental width of single, and often multiple, inverted-Vs.

We can relate this width to the latitudinal width $L_{x}$ first proposed by Lyons (1980). Assuming that large parallel currents are generated in regions of discontinuities in the convection electric field $(\nabla \cdot E<0)$ Lyons solved Eq. (2) by taking into account the Ohm's relationship between the field-aligned current density $j_{\|}$and the total field-aligned potential $V_{\|}$, $j_{\|} \approx j_{t h}+j_{t h} \cdot e V_{\|} / T_{e}$ where $T_{e}$ is the electron temperature and $j_{\text {th }}$ the thermal current given by $j_{\text {th }}=e n\left(T_{e} / 2 \pi m\right)^{1 / 2}$ (Lemaire and Sherer, 1974; Anto- 
nova and Tverskoy, 1975; Lyons, 1980). In that case $L_{x}$ is evaluated to be in the order of, $L_{x} \approx\left(\Sigma_{P} / Q\right)^{1 / 2}$, where $Q=j_{t h} / T_{e}$ is the field-aligned conductance. For $Q \sim 5 \times 10^{-10} \Omega^{-1} \mathrm{~m}^{-2}$ (Bosqued et al., 1986) and $\Sigma_{P} \sim 10 \mathrm{~S}$, we obtain $L_{x} \sim 200 \mathrm{~km}$, i.e. also within the order of the latitudinal width of inverted-Vs.

Thus, both scaling determinations give approximately the same latitudinal width, determined by the ionospheric conductivity and by the electron and ion plasma sheet properties: $n_{e}, T_{e}$, and $T_{i}$. Therefore, this scale of $100-200 \mathrm{~km}$ in latitude (at ionospheric altitude), can be considered as a natural scale for the magnetosphericionospheric coupling through field-aligned currents. Both approaches were mixed in the original Tverskoy paper (Tverskoy, 1982) and later by Antonova and Stepanova (1988). Originally, Tverskoy (1982) showed that his simple model, which assumes no parallel potential, as in Antonova et al. (1998), is only valid for single or double inverted-Vs, and that important modifications in the $\Gamma$ parameter value are introduced by taking account the presence of a field-aligned potential drop above inverted-Vs. In Tveskoy's model, it was shown that the basic criteria for the appearance of multiple oscillations and the resulting of multiple structures (three or more) is the condition:

$\alpha \cdot \frac{j^{\max }}{j_{t h}} \cdot \frac{T_{e}}{T_{i}}>1$

with $\alpha \sim 1$. In this case, if $T_{i} \sim 3 \mathrm{keV}$ and $T_{e} \sim 0.5 \mathrm{keV}$, the maximum field-aligned current must be sufficiently high, $\sim 6 . j_{t h}$ for the condition to be fulfilled. However statistical analysis (of the dependence of the occurrence of multiple structures on the maximum current density) remained relatively inclusive even though a density higher than $3 \times 10^{-6} \mathrm{~A} / \mathrm{m}^{2}$ favours highly structured events. As stressed by Tverskoy (1982) the differential equation describing the instability of a given (nonstratified) current distribution is much more complicated than Eq. (6), leading to a more complex $\Gamma$ expression; rough evaluation leads to $\Gamma$ values less than obtained from the simplest expression for a given set of substructures. This effect is clearly found in the statistical analysis, as shown in Fig. 6.

Summarizing, from the most relevant results obtained during this statistical analysis we may infer that the multiple structures currently observed may be seen to result from a natural instability of the current system coupling the ionosphere to the magnetosphere. They are possibly related to oscillations of the magnetospheric potential (or the pressure gradients) on a scale of $\sim 1 R_{E}$ in the near-Earth plasma sheet. These oscillations, as postulated in Tverskoy's model tested here, may naturally result when the initial pressure gradients needed to generate a large-scale field-aligned current have a sufficiently large equatorial scale, larger than one $R_{E}$.

Our findings can be related to previous results obtained using the same A3 database. As shown by Bosqued et al. (1993), the most intense discrete arcs or inverted-Vs detected by A3 (detected regardless of the level of magnetic activity) are often located in, or equatorward of, a region where the ion precipitation minimizes ("ion gap"). Ashour-Abdalla et al. (1992) and Bosqued et al. (1995), using numerical simulations, have interpreted this ion "gap"-electron arc association as the signature of a strong enhancement of the equatorial cross-tail current due to the non-adiabatic duskward drift of the ions near the ion "wall". Strong azimuthal pressure gradients are set up and can lead to strong FACs according to the Tverskoy mechanism discussed here. Moreover, Ashour-Abdalla et al. (1992) have suggested that this non-adiabatic escape is significant in removing the pressure built up in the near-Earth region. More recently Galperin and Bosqued (1998, 1999) have computed the FAC intensity and profile generated by azimuthal pressure gradients (coincident with the appearance of a minimum in the $B_{z}$ radial profile) and demonstrated a good agreement with A3 observations in case of a single electron arc. Clearly, even small fluctuations in the plasma pressure gradients like those suggested by Tverskoy (1982) are quite probable and will be strongly amplified by the Tverskoy mechanism to produce considerable amplitude variations and splitting in the generated FAC structures.

We did not attempt to infer an equatorial pressure profile from auroral ion data, as done by Wing and Newell $(1998,2000)$ from low-altitude DMSP data. Although this evaluation, which produces interesting pressure maps in the near-Earth plasma sheet, is quite feasible to obtain from A3 data assuming plasma sheet ions are nearly isotropic, it will give only rough profiles ( $\sim 1 R_{E}$ resolution) when mapped down to the equatorial plane. Pressure evaluation for inverted-Vs where the ion flux is sufficient to obtain pressure profiles at the best available space resolution $(\sim 10-15 \mathrm{~km}$ in the auroral zone), is deferred to another paper.

We close by concluding that the total width of the current auroral band or, in the equatorial plane at 10-15 $R_{E}$ downtail, the scale of radial pressure gradients and their possible oscillations on a spatial scale of $\leq 1 R_{E}$, seem to be predominant in determining the scale of the mapped ionospheric FAC sheets and their observed splitting on a scale of $100-200 \mathrm{~km}$ at ionospheric levels. How this mechanism operates in splitting auroral structures during substorm periods will be the topic of a future paper. Nevertheless, direct verification should be searched for in the equatorial near-Earth plasma sheet. The forthcoming CLUSTER-2 mission of ESA will be able to explore this region at least at the end of the mission when the four satellites will be well separated in space. At this time temporal and spatial variations will be well differentiated and the crucial radial and azimuthal plasma pressure gradients will be able to be calculated.

Acknowledgements. The authors would like to thank the referees who made many constructive comments. The ARCAD(A3)/ AUREOL project was developed within the framework of the France-former USSR Cooperation for Space Studies. The authors acknowledge the Centre National d'Etudes Spatiales (CNES, France) for creating the A3 database and are especially grateful to Mrs. J. Cuvilo (CESR) and C. Guerin (CETP) for help in processing the A3 data. This work was made possible by the 
support of the ECOS-Sud/CONECYT program for France-Chile Scientific Cooperation, under grants C99U02/053FRA. This work was also supported by the INTAS grant 94-1485, the FONDECYT grant 1990132, the DICYT of the Universidad de Santiago de Chile grant 049931S, the RFBR grant 98-05-64508, and the Ministry of Education of Russia. The work of J.M.B. was in part supported by CNES under grant 071/97/CNES/6709.

Topical Editor G. Chanteur thanks two referees for their help in evaluating this paper.

\section{References}

Ahn, B.-H., An ionospheric conductance model based on ground magnetic disturbance, J. Geophys. Res., 103, 14 769-14 780, 1998.

Antonova, E. E., and B. A. Tverskoy, On the nature of inverted-V electron precipitation band and Harang discontinuity in the evening sector of the auroral ionosphere, Geomagn. Aeron., (English translation), 15, 105-111, 1975.

Antonova, E. E., and N. Y. Ganushkina, Azimuthal hot plasma pressure gradients and dawn-dusk electric field formation, J. Atmos. Terr. Phys., 59, 1343-1354, 1997.

Antonova, E. E., and M. V. Stepanova, About the role of adiabatic and non-adiabatic processes in the formation of the characteristics of discrete aurora (in Russian), in Morphology and physics of aurora, 59-62, Polar Geophysical Institute of the Academy of Science URSS, Apatity, 1988.

Antonova, E. E., V. I. Lazarev, M. V. Stepanova, and B. A. Tverskoy, The influence of self-consistent ionospheric conductivity on the field-aligned current stratification, Geomagn. Aeron., (English translation), 28, 16-21, 1988.

Antonova, E. E., V. I. Lazarev, M. V. Stepanova, B. A. Tverskoy, M. V. Teltzov, A. K. Kuzmin, S. I. Schkolnikova, and N. I. Isaev, Parameter of multiple inverted-V structures inferred from Intercosmos-Bulgaria-1300 satellite data, Geomagn. Aeron., (English translation), 31, 258-267, 1991.

Antonova, E. E., M. V. Stepanova, and M. V. Teltzov, Particle flux fluctuations and the low-frequency turbulence spectrum according to Interkosmos-Bulgria-1300 satellite data, Geomagn. Aeron., (English translation), 33, 183-189, 1993.

Antonova, E. E., M. V. Stepanova, M. V. Teltzov and B. A. Tverskoy, Multiple inverted V structures and hot plasma pressure gradient mechanism of plasma stratification, J. Geophys. Res., 103, 9317-9332, 1998.

Ashour-Abdalla, M., L. M. Zelenyi, J. M. Bosqued, V. Peroomian, Z. Whang, D. Schriver, and R. L. Richard, The formation of the wall region: consequences in the near Earth magnetotail, Geophys. Res. Lett., 19, 1739-1742, 1992.

Berthelier, J. J., F. Lefeuvre, M. M. Mogilevsky, O. A. Molchanov, Y. I. Galperin, J. F. Karczewski, R. Ney, G. Gogly, C. Guérin, M. Levêque, J.-M. Moreau, and F. X. Sené, Measurements of the VLF electric and magnetic components of waves and DC electric field on board the AUREOL-3 spacecraft: the TBFONCH experiment, Ann. Geophysicae, 38, 643-667, 1982.

Borovsky, J. E., Auroral arc thickness as predicted by various theories, J. Geophys. Res., 98, 6101-6138, 1993.

Bosqued, J. M., H. Barthe, J. Coutelier, J. Crasnier, J. Cuvilo, J. L. Medale, H. Reme, H. Sauvaud, and R. A. Kovrazhkin, The low energy electron and ion spectrometers on the AUREOL-3 satellite. The SPECTRO experiment, Ann. Geophysicae, 38, 567-582, 1982.

Bosqued, J. M., C. Maurel, J. A. Sauvaud, R. A. Kovrazhkin, and Y. I. Galperin, Observations of auroral electric inverted-V structures by the Aureol-3 satellite, Planet. Space Sci., 34, 255269, 1986

Bosqued, J. M., M. Ashour-Abdalla, M. El Alaoui, L. M. Zelenyi, and A. Berthelier, Aureol-3 observations of new boundaries in the auroral ion precipitation, Geophys. Res. Lett., 20, 12031206, 1993.
Bosqued, J. M., M. Ashour-Abdalla, V. Peroomian, R. L. Richard, and L. M. Zelenyi, Auroral precipitation patterns: Observations and 3D simulations, in Substorms-2, Second International Conference on Substorms, Eds. Kan, J. R., J. D. Craven, and S.-I. Akasofu, 365-372, University of Alaska, Fairbanks, Alaska, 1995.

Boström, R., Mechanisms for driving Birkeland currents, in Physics of the hot plasma in the magnetosphere, Eds. Hultqvist, B., and L. Stenflo, Plenum, NY, 341-365, 1975.

Chiu, Y. T. and M. Schultz, Self-consistent particle and parallel electrostatic field distributions in the magnetosphere-ionosphere auroral region, J. Geophys. Res., 83, 629-642, 1978.

Chiu, Y. T., J. M. Cornwall, and M. Schultz, Effects of auroralparticle anisotropies and mirror forces on high-latitude electric fields, in Physics of auroral arc formation, AGU Geophysical Monograph, 25, 234-244, 1981.

Chiu, Y. T., J. M. Cornwall, F. J. Fennell, D. J. Gorney, and P. F. Mizera, Auroral plasmas in the evening sector: Satellite observations and theoretical interpretations, Space Sci. Rev., 35, 211-257, 1983.

Christon, S. P., L. A. Frank, C. Y. Huang, D. C. Mitchell, and D. J. Williams, Spectral characteristics of plasma sheet ion and electron populations during disturbed geomagnetic conditions, J. Geophys. Res., 96, 1-22, 1991.

Evans D. S., Precipitating electron fluxes formed by a magnetic field-aligned potential difference, J. Geophys. Res., 79, 28532863, 1974.

Fennell, J. F., D. J. Gorney, and P. F. Mizera, Auroral particle distribution functions and their relationship to inverted Vs and auroral arcs, in Physics of auroral arc formation, AGU Geophysical Monograph, 25, 91-102, 1981.

Frank, L. A., and K. L. Ackerson, Observations of charged particle precipitation into the auroral zone, J. Geophys. Res., 76, 3612$3643,1971$.

Fridman, M., and J. Lemaire, Relationship between auroral electron fluxes and field-aligned electric potential differences, J. Geophys. Res., 85, 664-670, 1980.

Galperin, Y. I., Stable auroral arcs: observations and models, in Substorms 2, Eds. Akasofu, S.-I., and J. Craven, University of Alaska, Fairbanks, USA, 383-390, 1994.

Galperin, Y. I., and J. M. Bosqued, A new mechanism of substorm onset or local auroral activation, Cosmic Res., 36, 109-117, 1998.

Galperin, Y. I., and J. M. Bosqued, Stationary magnetospheric convection on November 24, 1981. 1. A case study of "pressure gradient/minimum-B" auroral arc generation, Ann. Geophysicae, 17, 358-374, 1999.

Galperin, Y. I., A. V. Volosevich, and L. M. Zelenyi, Pressure gradient structures in the tail neutral sheet as "Roots of the Arcs" with some effects of stochasticity, Geophys. Res. Lett., 19, 2163-2166, 1992.

Gjerloev, J. W., and R. A. Hoffman, Height-integrated conductivity in auroral substorms, 1. Data, J. Geophys. Res., 105, 215-226, 2000.

Grad, H., Some new variational properties of hydromagnetic equilibria, Phys. Fluids, 7, 1283-1292, 1964.

Hardy, D. A., M. S. Gussenhoven, R. Raistrick, and W. J. McNeil, Statistical and functional representations of the pattern of auroral energy flux, number flux and conductivity, J. Geophys. Res., 92, 12 275-12 294, 1987.

Harel, M., R. A. Wolf, P. H. Reiff, R. W. Spiro, W. J. Burke, F. J. Rich, and M. Smiddy, Quantitative simulation of a magnetospheric substorm, 1. Model logic and overview, J. Geophys. Res., 86, 2217-2241, 1981.

Heelis, R. A., W. B. Hanson, and J. L. Burch, AE-C observations of electric fields around auroral arcs, in Physics of auroral arc formation, AGU Geophysical Monograph, 25, 154-163, 1981.

Heikkila, W. I., Satellite observations of soft particle fluxes in the auroral zone, Nature, 225, 369-370, 1970.

Heinemann, M., Representations of currents and magnetic fields in anisotropic magnetohydrostatic plasma, J. Geophys. Res., 95, 7789-7797, 1990. 
Heinemann, M., and D. H. Pontius, Representation of currents and magnetic fields in anisotropic magnetohydrostatic plasma 2. General theory and examples, J. Geophys. Res., 96, 17 60517 626, 1991.

Hoffman, R. A., M. Sugiura, and N. C. Maynard, Current carriers for the field-aligned current system, Adv. Space Res., 5, 109-126, 1985.

Huang, C. Y., and L. A. Frank, A statistical survey of central plasma sheet, J. Geophys. Res., 99, 83-95, 1994.

Iijima, T., T. A. Potemra, and L. J. Zanetti, Contribution of pressure gradients to the generation of dawnside region 1 and region 2 currents, J. Geophys. Res., 102, 27 069-27 082, 1997.

Johnson, M. L., J. S. Murphree, G. T. Marklund, and T. Karlsson, Progress on relating optical auroral forms and electric field patterns, J. Geophys. Res., 103, 4271-4284, 1998.

Klumpar, D. M., Tranversely accelerated ions in auroral arcs, in Physics of auroral arc formation, AGU Geophysical Monograph, 25, 122-128, 1981.

Knight, L. R., Parallel electric fields, Planet. Space. Sci., 21, 741$750,1973$.

Lemaire, J., and M. Sherer, ionosphere-plasma sheet field-aligned currents and parallel electric fields, Planet. Space Sci., 22, 14851490, 1974.

Lin, C. S., and R. A. Hoffman, Observations of inverted-V electron precipitation, Space Sci. Rev., 33, 415-457, 1982.

Lin, C. S., J. N. Barfield, J. L. Burch, and J. D. Winningham, Near conjugate observations of inverted- $\mathrm{V}$ electron precipitation using DE-1 and DE-2, J. Geophys. Res., 90, 1669-1681, 1985.

Lyons, L. R., Generation of large-scale regions of auroral currents, electric potentials, and precipitation by the divergence of the convection electric field, J. Geophys. Res., 85, 17-24, 1980.

Lyons, L. R., The field-aligned current versus potential relation and auroral electrodynamics, in Physics of auroral arc formation, AGU Geophysical Monograph, 25, 252-259, 1981a.

Lyons, L. R., Discrete aurora as the direct result of an inferred, high altitude generating potential distribution, J. Geophys. Res., 86, 1-16, $1981 b$.

McFadden, J. P., C. W. Carlson, and R. E. Ergun, Microstructure of the auroral acceleration region as observed by FAST, J. Geophys. Res., 104, 14 453-14 480, 1999.
Pulliam, D. M., H. R. Anderson, K. Stammes, and M. H. Rees, Auroral electron acceleration and atmospheric interactions: (1) rocket-borne observations and (2) scattering calculations, J. Geophys. Res., 86, 2397-2404, 1981.

Robinson, R. M., and R. R. Vondrak, Measurements of $E$ region ionization and conductivity produced by solar illumination at high latitudes, J. Geophys. Res., 89, 3951-3956, 1984.

Robinson, R. M., R. R. Vondrak, K. Miller, T. Dabbs, and D. A. Hardy, On calculating ionospheric conductances from the flux and energy of precipitating electrons, J. Geophys. Res., 92, 2565-2569, 1987.

Spiro, R. W., P. H. Reiff, and L. J. Maher Jr., Precipitating electron energy flux and auroral zone conductivities - an empirical model, J. Geophys. Res., 87, 8215-8227, 1982.

Stasiewicz, K., and T. Potemra, Multiscale current structures observed by Freja, J. Geophys. Res., 103, 4315-4326, 1998.

Sugiura, M., T. Iyemori, R. A. Hoffman, N. C. Maynard, J. L. Burch, and J. D. Winningham, Relationship between field-aligned currents, electric fields and particle precipitation as observed by Dynamics Explorer-2, in Magnetospheric currents, AGU Geophysical Monograph, 28, 96, AGU, Washington, 1984.

Thieman, J. R., and R. A. Hoffman, Determination of inverted-V stability from Dynamics Explorer satellite data, J. Geophys. Res., 90, 3511-3516, 1985.

Tverskoy, B. A., Field-aligned currents in the magnetosphere, Geomagn. Aeron., (English translation), 22, 812-815, 1982a.

Tverskoy, B. A., Nature of homogeneous polar auroral arcs, Geomagn. Aeron., (English translation), 22, 794-799, 1982b.

Tverskoy, B. A., Magnetosphere-ionosphere interaction, Usp. Fiz. Nauk (in Russian), 4, 737-739, 1983.

Vasyliunas, V. M., Mathematical models of magnetospheric convection and its coupling to the ionosphere, in Particles and fields in the magnetosphere, Ed. B. M. McCormac, 60-71, D. Reidel, Hingham, Mass., 1990.

Wing, S., and P. T. Newell, Central plasma sheet ion properties as inferred from ionospheric observations, J. Geophys. Res., 103, 6785-6800, 1998.

Wing, S., and P. T. Newell, Quiet time plasma sheet ion pressure contribution to Birkeland currents, J. Geophys. Res., 105, 77937802, 2000. 